\title{
EDIFICATE
}

I Congreso de Escuelas de Edificación y Arquitectura Técnica de España València, 4 y 5 de noviembre de 2021

Escuela Técnica Superior de Ingeniería de Edificación

Universitat Politècnica de València

Doi: https://doi.org/10.4995/EDIFICATE2021.2021.13249

\section{Guía de recomendaciones para la Gestión y tratamiento de RESIDUOS generados por las medidas de prevención ante la COVID-19 en obras de construcción}

\section{Recommendations guide for the Management and treatment of WASTE generated by prevention measures against COVID-19 in construction sites}

\footnotetext{
Francisco J. Sánchez Medrano ${ }^{a}$, Salvador Aledo Guerao ${ }^{b}$ y Jesús H. Alcañiz Martínez ${ }^{\mathrm{c}}$ aDr. Arquitecto, profesor UCAM, fjsanchez@ucam.edu, bDr. Ingeniero de Edificación, profesor UCAM, saledo@ucam.edu y Dr. Ingeniero de Edificación, profesor UCAM, jalcaniz@ucam.edu.
}

\begin{abstract}
The COVID-19 pandemic crisis has affected all areas of society, from production to classrooms. As teachers and researchers, and given the emergence of new elements of disease prevention, and therefore new waste in construction, we have been participants in the profusion of news, rules and recommendations that have fallen on all of us.

The need to live with the health threat, and to continue economic activity, required a clear and concise structure for the management of these wastes so that they do not become another source of added risk.

We have worked for several months in collaboration with more than 20 construction companies, to give an answer in the form of a Guide of recommendations for the management of these elements, also involving the students of Health and Safety and Construction Process Management, of the importance of incorporating changes into the development of a work, because the context is an inseparable part of our task.
\end{abstract}

Keywords: Health and Safety, Risk prevention, incorporation of new challenges, process disturbances 


\section{Resumen}

La crisis de la pandemia del COVID-19 ha afectado a todos los ámbitos de la sociedad, desde la producción hasta las aulas. Como docentes e investigadores, $y$ ante el surgimiento de nuevos elementos de prevención de la enfermadad, y por tanto, de nuevos residuos en la construcción, hemos sido partícipes de la profusión de noticias, reglas y recomendaciones que han caído sobre todos nosotros.

La necesidad de convivir con la amenaza sanitaria, y de proseguir la actividad económica, precisaba de una estructura clara y concisa del manejo de estos residuos para que no se conviertan en una fuente más de riesgo añadida.

Hemos trabajado varios meses en colaboración con más de 20 empresas constructoras, para dar una respuesta en forma de Guía de recomendaciones para la gestión de estos elementos, haciendo partícipes también a los alumnos de Seguridad y Salud y Gestión del Proceso constructivo, de la importancia de incorporar los cambios al desarrollo de una obra, porque el contexto forma parte inseparable de nuestro trabajo.

Palabras clave: Seguridad y Salud, Prevención de riesgos, incorporación de nuevos retos, alteraciones del proceso. 


\section{Introducción}

La declaración de pandemia de COVID-19 el 11 de marzo de 2020 por la Organización Mundial de la Salud elevó la situación de emergencia sanitaria. En este contexto y tras la grave incidencia de la enfermedad en nuestro país, el 14 de marzo se declaró el estado de alarma por medio del Real Decreto 463/2020, tras el que se han aprobado sucesivas regulaciones en todos los sectores productivos, entre ellos el de la construcción.

Como consecuencia de ello, han sido numerosas las instituciones que han desarrollado recomendaciones y protocolos de actuación en materia de seguridad y salud en las obras de construcción con el objetivo de adaptar el sector a la situación sanitaria actual. Estas guías contienen, entre otras, medidas de distanciamiento de los trabajadores, pero apuntan también a la necesidad de extremar las condiciones de limpieza de los centros de trabajo y al uso de equipos de protección individual (EPI), en muchos casos desechables, cuando las condiciones del trabajo lo requieran

Ha aparecido en las obras de construcción, un nuevo tipo de residuo, relacionado con la prevención de la COVID-19, cuya gestión, almacenamiento y reciclaje o destrucción requiere de unos ciertos protocolos de actuación. Se trata de una alteración contextual que afecta a la Seguridad y Salud en obra e introduce un nuevo factor en la gestión del proceso constructivo. Para que estas alteraciones fueran rápidamente asimiladas en obra, hemos procurado que estas nuevas indicaciones se expusieran de forma concisa y práctica en un documento con formato de Guía.

\section{Objetivos}

El grupo de investigación ARIES (Arquitectura, Ingeniería de Edificación y Sostenibilidad), está constituido por un conjunto de técnicos del ámbito de la construcción, con el objetivo principal de buscar soluciones sostenibles a los problemas que se plantean en la edificación del s. XXI.

En este sentido, y también como docentes, la presencia en la sociedad de la COVID-19 ha supuesto un importante reto para adaptar las medidas de prevención y delimitación de riesgos, que ha merecido nuestra atención, centrándonos en el tratamiento de un nuevo conjunto de residuos añadidos a los tradicionales del sector.

Con el patrocinio de la UCAM, en convocatoria de financiación propia, hemos estado trabajando durante varios meses, en contacto con más de 20 empresas constructoras, para extractar la abundante información vertida desde distintos organismos, y aplicar una metodología de funcionamiento que resuma en un decálogo los procedimientos a seguir con este nuevo peligro biológico con el que deberemos acompasar nuestras tareas.

Como no se ha interrumpido la docencia, sino que se ha trasladado a una presencialidad adaptada a las circunstancias y al soporte digital, los avances del proyecto de investigación han sido compartidos con los alumnos de Seguridad y Salud y de las diversas materias de 
Gestión del Proceso Constructivo, a modo de ejemplo de la incorporación de elementos externos obligatorios y necesarios para la continuidad de los trabajos.

La responsabilidad sobre las medidas de salud, en este caso, opera como alteración imprevista en la organización de los tajos (distancias de seguridad), accesos de obra, incremento de bajas por enfermedad, provision de nuevos elementos EPI y generación de residuos. Cuestión real e insoslayable que supera la virtualidad de ejemplos prácticos y obliga a una revisión de los supuestos económicos fundamentales de obra (plazo y precio).

\section{Desarrollo de la investigación}

Una obra de construcción es cualquier obra, pública o privada, en la que se efectúen trabajos de construcción o ingeniería civil cuya relación no exhaustiva figura en el anexo I del Real Decreto 1627/1997, de 24 de octubre, por el que se establecen disposiciones mínimas de seguridad y de salud en las obras de construcción.

En ellas, actúa una o varias empresas contratistas que actúan como titulares del centro de trabajo. Las medidas preventivas a aplicar respecto a la protección de los trabajadores frente a la COVID-19 en obra son, de forma general, las que indique el Ministerio de Sanidad o Ministerio de Trabajo para cualquier centro de trabajo (siendo el RD 463/2020 de aplicación, por tanto, en las obras de construcción).

El contratista, titular del centro de trabajo, deberá establecer un procedimiento de actuación en cada obra, siguiendo el "Procedimiento de actuación para los servicios de prevención de riesgos laborales frente a la exposición al SARS-CoV- 2", publicado por el Ministerio de Sanidad, o cualquier normativa análoga del ámbito territorial que sea pertinente.

El documento que se ha elaborado es el resultado de un Proyecto de Investigación del programa PMAFI-COVID 19, convocatoria de financiación propia de la Universidad Católica de Murcia, desarrollado por el Grupo de Investigación Arquitectura Ingeniería de Edificación y Sostenibilidad (ARIES); tiene carácter informativo y preventivo de carácter general, recogiéndose una selección no exhaustiva de recomendaciones y medidas de tipo sanitario e higiene en las obras.

Como se ha expuesto, su formulación se ha producido a lo largo del curso 20-21, y ha estado cimentado en una encuesta en la que han participado más 20 empresas constructoras $\mathrm{Ha}$ servido como ejemplo real en sesiones prácticas de alumnos de los últimos niveles de Ingeniería de Edificación/Arquitectura Técnica, trasladando las circunstancias impuestas al desarrollo de las obras, evaluando los efectos producidos, y reafirmando la conveniencia de dotar con un sistema ordenado y práctico de medidas a seguir. 


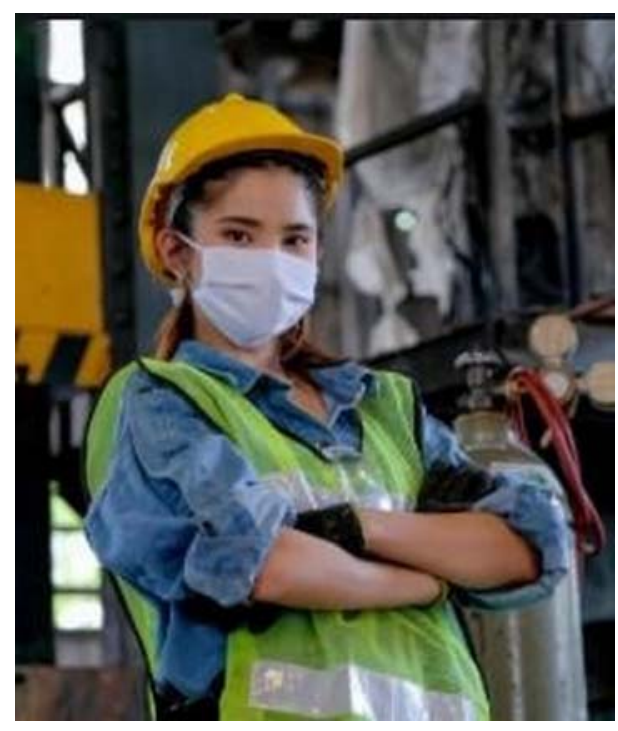

Fig. 1 Trabajadora con mascarilla de protección

\section{Resultados/conclusiones: Gestión de los nuevos residuos en la obra}

(Mascarillas, guantes, pantallas faciales, cubrezapatos, felpudos desinfectantes, toallas de papel, pañuelos, botes de desinfección, etc.)

1. La gestión de los residuos ordinarios continuará realizándose del modo habitual, respetando los protocolos de separación de residuos.

2. Se recomienda que los pañuelos desechables que el personal emplee para el secado de manos o para el cumplimiento de la "etiqueta respiratoria" sean desechados en papeleras o contenedores protegidos con tapa y, a ser posible, accionados por pedal.


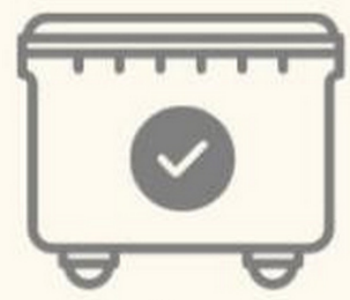

Fracción resto

Fig. 2 Esquema de depósito de protecciones sin contagio 

prevención ante la COVID-19 en obras de construcción. Recommendations guide for the Management and treatment of WASTE generated by prevention measures against COVID-19 in construction sites

3. Hay que facilitar en las zonas comunes (Vestuarios, aseos), y en las proximidades de los puestos de trabajo geles hidroalcohólicos, toallas de papel desechables y papeleras de pedal con tapa para depositar residuos.

4. Para una mejor toma de responsabilidad se recomienda que junto a los contenedores se dispongan expendedores de bolsas desechables para depositar en ellas los residuos personales envueltos.

5. Todo material de higiene personal (pañuelos desechables, mascarillas, guantes de látex, etc.), debe depositarse en la fracción resto (agrupación de residuos de origen doméstico que se obtiene una vez efectuadas las recogidas separadas).

6. El contenedor o papelera tipo para este tipo de fracción es el de COLOR GRIS, y deberá ser sometido a un procedimiento de limpieza y desinfección, tanto por dentro como por fuera, cada vez que se eliminen la bolsa con los residuos.

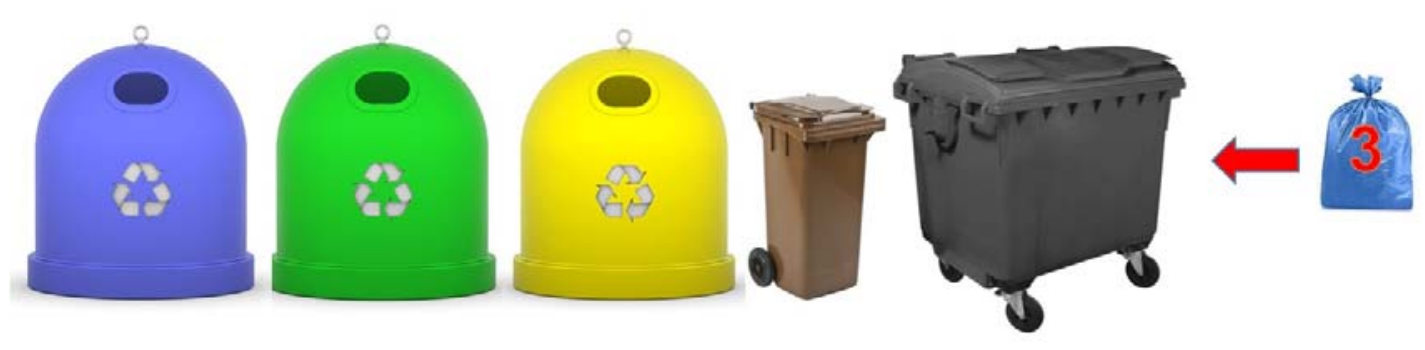

Fig. 3 Contenedor a utilizar

7. Para desinfectar el material utilizado para la limpieza (bayetas, trapos, fregonas, etc., si no son desechables) se deben sumergir en agua con un poco de lejía durante 10 minutos, aclarar y dejar secar bien.

8. No existe obligación legal de realizar la desinfección por una empresa autorizada. Se podrá realizar la desinfección por el propio personal de la empresa y utilizando detergentes habituales.

9. Sólo en el caso de que para la desinfección se decida utilizar productos virucidas cuya aplicación requiera de personal profesional especializado, las empresas que realizan estos tratamientos deberán ser empresas de servicios que estén expresamente registradas para ello en el Registro Oficial de Establecimientos y Servicios Biocidas de las Comunidades Autónomas.

10. En caso de que un trabajador presente síntomas mientras se encuentre en su puesto de trabajo, será preciso aislar el contenedor donde haya depositado pañuelos u otros 
productos usados. Esa bolsa de basura deberá ser extraída y colocada en una segunda bolsa de basura, con cierre, para su depósito en la fracción resto.

En algunas CCAA estos casos pueden ser tratados como residuos sanitarios tipo III, cuando se comprueba que se trata de un caso positivo de COVID-19. Con carácter general, los contenedores de fracción resto vacíos y las ubicaciones de los mismos en el propio centro de trabajo, serán limpiados interior y/o exteriormente y baldeados con productos desinfectantes con la mayor frecuencia posible, debiendo establecerse al efecto protocolos de limpieza y desinfección de estas zonas.



Fig. 4 Esquema de depósito de protecciones existiendo contagio

\section{RECOMENDACIONES SOBRE USO DE EQUIPOS DE PROTECIÓN INDIVIDUAL}

- En la medida de lo posible, evitar compartir los equipos de protección y, en ningún caso, compartir equipos de protección respiratoria.

- Mascarillas: la mayoría de estos equipos vienen marcados con las letras "NR" (no reutilizable), por lo que lo más recomendable es desecharlas tras su uso. En el caso de que el marcado de la misma sea "R" (reutilizable) es recomendable, tras su uso, dejarla en cuarentena durante 72 horas, ya que determinados métodos de lavado y desinfección pueden alterar su estructura filtrante, por lo que pierden eficacia. En algunos casos es posible sumergir la mascarilla en soluciones de hipoclorito sódico. Para estos casos, consultar en el libro de instrucciones o con el fabricante.

- Guantes: el sistema de desinfección variará en función del material del mismo.

- Látex o nitrilo: se desecharán siempre tras cada uso.

- Cuero: generalmente, no soportan el lavado, por lo que se pulverizarán con una solución hidroalcohólica al $70 \%$ dejando secar al aire. Otra opción sería mediante el calor seco.

- Goma o plástico: es necesario consultar el manual de instrucciones del equipo, aunque, en general, podrán limpiarse con una solución hidroalcohólica al $70 \%$.

- Textil: en general, se podrán lavar conforme se ha descrito anteriormente, consultar previamente instrucciones del fabricante. 

prevención ante la COVID-19 en obras de construcción. Recommendations guide for the Management and treatment of WASTE generated by prevention measures against COVID-19 in construction sites

- Calzado: se podrán desinfectar con una solución hidroalcohólica al 70\%.

- Arnés: no se deben utilizar productos químicos, ya que pueden deteriorar las fibras textiles y materiales plásticos. Un arnés que haya sido utilizado se dejará en cuarentena antes de su utilización por otro trabajador. Para determinar el tiempo que debe mantenerse sin uso, se tendrán en cuenta los materiales que componen el arnés, por ejemplo, en los metales puede permanecer hasta 96 horas, tiempo para determinar el periodo de cuarentena del arnés. Para su desinfección mediante cualquier otro procedimiento (lavado con agua caliente, uso de productos químicos, etc.), se deberá consultar con el fabricante.

- Gafas o pantallas faciales: se pueden limpiar con toallitas empapadas en desinfectantes y, tras su total secado, aclarar con una segunda toallita empapada con agua el exceso de producto restante para no entorpecer la visión. Como solución desinfectante puede usarse, de forma general, una solución hidroalcohólica.

\section{LIMPIEZA, DESINFECCIÓN E HIGIENE}

Practique la higiene de las manos, la higiene respiratoria y las normas de higiene para la tos, así como la limpieza y desinfección.

- Use agua y jabón para lavarse frecuentemente las manos (por al menos 20 segundos), y toallas de papel para secárselas.

- Use un desinfectante de manos con al menos un $60 \%$ de alcohol a menudo, cuando no sea fácil lavarse las manos.

- Cúbrase la nariz y la boca con un pañuelo desechable o la parte de arriba de la manga al toser o estornudar.

- Cuando sea posible, evite compartir objetos y equipos con otros empleados, incluidos los teléfonos, los escritorios u otras mesas de trabajo, herramientas y equipos.

- Lave y desinfecte los objetos y las superficies que se tocan frecuentemente, como herramientas compartidas, máquinas, vehículos y otros equipos, barandas, escaleras, manijas de puertas, y baños portátiles. Las superficies que estén visiblemente sucias deben limpiarse con agua y jabón antes de desinfectarlas.

\section{DISTANCIAMIENTO SOCIAL Y MASCARILLAS}

Siga las guías de distanciamiento social y uso de mascarillas:

- Mantenga al menos 2 metros de distancia (aproximadamente el largo de 2 brazos) entre trabajadores, siempre que sea posible.

- Mantenga el distanciamiento social durante los descansos y cuando coma.

- Evite las áreas cerradas, como casas móviles y espacios reducidos. 
- Póngase una mascarilla en los lugares de trabajo, especialmente cuando sea difícil mantener el distanciamiento social.

- Póngase una mascarilla que le cubra la nariz y la boca para lograr la máxima protección (no se ponga la mascarilla alrededor del cuello o sobre la frente).

- No toque la parte de afuera de la mascarilla cuando la tenga puesta; si lo hace, lávese las manos o use un desinfectante de manos con al menos un $60 \%$ de alcohol para desinfectarse.

- Tenga cuidado de no tocarse los ojos, la nariz ni la boca al quitarse la mascarilla, y lávese las manos de inmediato después de hacerlo, a menos de que se las haya lavado recientemente

\section{Referencias}

Ley 31/1995, de 8 de noviembre, de Prevención de Riesgos Laborales.

Real Decreto 646/2020, de 7 de julio, por el que se regula la eliminación de residuos mediante depósito en vertedero.

Real Decreto 773/1997, de 30 de mayo, sobre disposiciones mínimas de seguridad y salud relativas a la utilización por los trabajadores de equipos de protección individual.

Aparejadores Madrid: Resumen de los principales aspectos relacionados con la actuación de la INSPECCIÓN DE TRABAJO Y SEGURIDAD SOCIAL respecto de la VIGILANCIA del cumplimiento de las MEDIDAS PREVISTAS en el RD-Ley 21/2020, y analizadas en el CRITERIO TÉCNICO 103/2020 "Sobre actuaciones de la Inspección de Trabajo y seguridad social relativas a la habilitación contenida en el real decreto-ley 21/2020, de 9 de junio, en relación con las medidas de prevención e higiene para hacer frente a la crisis sanitaria ocasionada por la Covid-19 en los centros de trabajo

AA VV (2020): Estrategia de arquitectura y construcción sostenible. Consejería de Fomento e Infraestructuras. Di. Gral. De Territorio y Arquitectura. Murcia

AA VV (2016): Guía sobre gestión de residuos de construcción y demolición. AEDED.

Asociación Española de Empresarios de Demolición.

ADPR (Asociación de Descontaminación de Residuos Peligrosos) (2011): Residuos peligrosos en la Construcción y en la obra pública. Interempresas Media.

CDC (USA) Centros para el Control y la Prevención de Enfermedades.

CDCINFO: 1-800-CDC-INFO (1-800-232-4636) i TTY; 1-888-232-6348. Web: cdc.gov/info

prevencionygestionderesiduos_tcm30-509671

https://apps.who.int/iris/bitstream/handle/10665/331856/WHO-2019-nCoV 
Guía de recomendaciones para la Gestión y tratamiento de RESIDUOS generados por las medidas de prevención ante la COVID-19 en obras de construcción. Recommendations guide for the Management and treatment of WASTE generated by prevention measures against COVID-19 in construction sites

Food_Safety-2020.1-spa.pdf

CPWR The Center for Construction Reserach and Training

CSCAE. 09-04-2020: Recomendaciones de seguridad en obras de construcción por la crisis del Covid-19

Ecorys (2016): Protocolo de gestión de residuos de la construcción y demolición en la UE. Comisión Europea. Dir. Gral. Mercado Interior, Industria, Emprendimiento y Pymes.

Guía de medidas frente al CORONAVIRUS en obras de construcción (CNC, UGT, CCOO)

ICASST Orientaciones preventivas frente al covid-19 en obras de construcción

Instituto Nacional de Seguridad y Salud en el Trabajo. Prevención de riesgos laborales vs. COVID-19: compendio no exhaustivo de fuentes de información. INSST 2020. Disponible en: www.insst.es

Instituto de Salud Pública y Laboral de Navarra. Web oficial sobre el coronavirus https://coronavirus.navarra.es/es/

Instituto Valencià de Seguretat i Salut en el Treball: Gestión de residuos. medidas técnicas y organizativas preventivas frente a la exposición al coronavirus (Sars-Cov-2)

LEITE, Wolfgang (1981): la Química y la protección del medio ambiente. Ed. Paraninfo. Madrid.

Listado de virucidas autorizados en España para uso ambiental (PT2), industria alimentaria (PT4) e higiene humana (PT1) 26.03.2020. Ministerio de Sanidad Buenas prácticas en el centro de trabajo (PDF) Gobierno de España

Ministerio para la Transición Ecológica y el reto demográfico: Residuos Sanitarios.

https://www.miteco.gob.es/es/calidad-y-evaluacion-ambiental/temas/prevencion-y-gestionresiduos/flujos/residuos-sanitarios/

Ministerio de Sanidad (2020). Procedimiento de actuación para los servicios de prevención de riesgos laborales frente a la exposición al SARS-CoV-2, de 8 de abril de 2020.

Ministerio de Sanidad (2020). Procedimiento de actuación para los servicios de prevención de riesgos laborales frente a la exposición al nuevo coronavirus (SARS-COV-2).

Ministerio de Sanidad (2020). Estrategia de detección precoz, vigilancia y control de COVID-19.

Ministerio de Sanidad (2020). Medidas higiénicas para la prevención de contagios del COVID-19.

Ministerio de Sanidad (2020). Guía de buenas prácticas en centros de trabajo para prevenir los contagios del COVID19. 
OTERO DEL PERAL, Luis R. (1992): Residuos sólidos urbanos. Secretaría de Estado para las Políticas del Agua y el Medio Ambiente. Ministerio de obras Públicas y Transportes. Madrid

Protocolo Para la Gestion de Residuos Sanitarios Generados en los Centros Dependientes del Sescam.

https://www.chospab.es/enfermeria/Documentos/Protocolo_Residuos.pdf

PÉREZ DE LAMA, J. (2008): Ecología metropolitana: Arquitectura bioclimática, desarrollo sostenible, ecosofía. Composición Arquitectónica. Universidad de Sevilla.

http://htca.us.es/blogs/perezdelama

PÉREZ GUERRA, Alfonso (1991): Planificación de la Seguridad e Higiene en el Trabajo en Construcción y Conservación de Edificios. IFAS. Sección España. Barcelona

Servicio de PRL de la Generalitat www.prevencio.gva.es

VILLORIA SÁEZ, Paola (2014): Sistema de gestión de residuos de construcción y demolición en obras de edificación residencial. Buenas prácticas en la ejecución de obra. Tesis Doctoral. Univ. Politécnica de Madrid.

WHO-2019-nCoV-Workplace-actions-Policy-brief-2021.1-eng 